\section{CPS-128 LIVER TRANSPLANT AND DIABETES MELLITUS}

M Vera Cabrera*, I Plasencia García, A Ferrer Machín, I Gonzalez García, T Betancor García L Díaz Díaz, R Mesa Exposito, S Hernandez Rojas, K Alvarez Tosco, C Perez Martin, J Merino Alonso. Hospital Nuestra Señora La Candelaria, Hospital Pharmacy, Santa Cruz of Tenerife, Spain

10.1136/ejhpharm-2020-eahpconf.229

Background and importance Transplanted patients are at risk of developing post-transplant diabetes as a metabolic complication of immunosuppressive therapy, which results in greater therapeutic complexity.

Aim and objectives To evaluate the percentage of liver transplant patients with diabetes mellitus and the evolution of diabetes after 1 year of transplantation.

Material and methods An observational, descriptive, retrospective study was conducted in liver transplant patients during the period January 2013 to October 2018. The main variables included were the presence or absence of diabetes in the pretransplant period, immediate post-transplant period and 1 year after the transplant was performed; and the need for insulin use in each of these periods. All patients who died before 1 year after liver transplantation were excluded from the study.

Results During the study period, a total of 179 patients were included, $73.2 \%$ were men. Mean age of the patients was $54.8 \pm 9.6$ years.

Of the 179 patients, $69.8 \% \quad(n=125)$ were not diabetic before transplantation, $42.4 \%$ developed post-transplant diabetes $(n=53)$ and all were insulin dependent. One year after the transplant, $43.4 \% \quad(n=23)$ did not need to continue using insulin. Of the $30.2 \%(n=54)$ of patients who were diabetic prior to transplantation, $46.3 \% \quad(n=25)$ were not insulin dependent. In $88 \%$ of these patients $(n=22)$, post-transplant insulin therapy was necessary and $84 \%$ of patients $(n=21)$ continued on insulin therapy 1 year after transplantation.

Conclusion and relevance Liver transplanted patients had a high prevalence of diabetes requiring administration of insulin, which adds greater complexity to the treatment. Post-transplant diabetes is a metabolic complication that appears in the post-transplant period as a result of immunosuppressive treatment in both previously diabetic and non-diabetic patients. Non-insulin dependent diabetic patients are more likely to require insulin 1 year after transplant.

\section{REFERENCES AND/OR ACKNOWLEDGEMENTS}

No conflict of interest.

\section{CPS-129 PILOT STUDY TO SURVEY THE ATTITUDE, SUPPLEMENT USE AND STORAGE CONDITIONS OF DRUG PRODUCTS AMONG PATIENTS RECEIVING BIOLOGICAL THERAPY}

RG Vida*, A Somogyi-Végh, M Mayer, D Kiss, N Schaadt, R Rajj, B Molnár, L Botz. University of Pécs Faculty of Pharmacy, Department of Pharmaceutics and Central Clinical Pharmacy, Pécs, Hungary

\subsection{6/ejhpharm-2020-eahpconf.230}

Background and importance Biological therapies have recently become the cornerstone for the treatment of several dermatological and rheumatological diseases. As compensation for the extra workload associated with it, the system of itemised reimbursement of these products provides hospital pharmacists with a deeper insight and closer involvement in the therapy.
Aim and objectives Our aim was to assess the factors potentially affecting the effectiveness and safety of the therapy, and to become familiar with patient opinions on the drug supply, current form of dispensing and information received.

Material and methods Data were collected through structured personal interviews and review of the medical records. Twenty-six dermatological and 37 rheumatological patients were interviewed at the point of dispensing of the biologics. The survey focused on concomitant drugs and supplementary products (dietary supplements, herbal remedies, etc), patient opinions, and experience and knowledge of biological therapy. In the case of an additional 28 participants, storage conditions at the patients' homes were assessed with a Testo 184 T3 temperature data logger.

Results The 32 women and 31 men who completed the survey had been receiving biological therapy for an average of 6.5 years. There was a switch between biological agents in 21 patients and therapy had been changed twice in 10 patients. The average number of prescribed medicines and supplementary products were $6.6(1-24)$ and $2.3(1-8)$, respectively. A total of 33 patients $(52.4 \%)$ used at least one supplementary product. We identified two main topics that patients wanted more information on: side effects and available alternatives in the event of therapeutic failure. Of 28 temperature logs, only 3 remained between 2 and $8^{\circ} \mathrm{C}$.

Conclusion and relevance Biological therapies have revolutionised the clinical care of many diseases but because of their costs, it is essential to identify factors that influence therapeutic outcomes. Also, hospital and clinical pharmacists have the opportunity and competence to contribute to the optimisation of therapy. As data on home storage conditions, drug-drug and drug-supplement interactions with monoclonal antibodies are lacking, this pilot study can be the first step towards understanding the importance of these factors and their effect on the safety and effectiveness of the therapy.

\section{REFERENCES AND/OR ACKNOWLEDGEMENTS}

No conflict of interest.

\section{CPS-130 ONE IN A MILLION: A TNF RECEPTOR-1 ASSOCIATED SYNDROME RESISTANT TO ANTI-TNF-ALPHA THERAPY}

${ }^{1} B C$ Sánchez Lerma*, ${ }^{2} M A ́$ Pérez Cruz, ${ }^{1} Y$ Hernández Gago, ${ }^{1} M P$ González Joyanes, ${ }^{1}$ AL García Silva, ${ }^{1}$ MA Velaz Suarez. ${ }^{1}$ Hospital Pharmacist, Hospital Pharmacy Department. Complejo Hospitalario Universitario Insular Materno Infantil, Las Palmas De Gran Canaria, Spain; ${ }^{2}$ Hospital Pharmacist, Hospital Pharmacy, Complejo Hospitalario Universitario Insular Materno Infantil, Las Palmas De Gran Canaria, Spain

\subsection{6/ejhpharm-2020-eahpconf.231}

Background and importance Tumour necrosis factor receptor associated periodic syndrome (TRAPS) is a rare disorder with a prevalence of approximately 1 per million. The goal of therapy is prevention of recurrent symptoms and normalisation of inflammatory markers. These patients also have an increased risk of developing amyloidosis. Clinical experience and extrapolation from other autoinflammatory disorders suggest that early institution of biologics can lowers this risk. Historically, antitumour necrosis factor (TNF) therapy (etanercept) was used for patients with frequent and/or severe recurrences and for those with TNF receptor-1 (TNFR1) gene mutations associated with a high risk of amyloidosis.

Aim and objectives To improve evidence about TRAPS refractory to anti-TNFs and its management. 
Material and methods We describe the case of a child affected by TRAPS and its pharmacotherapeutic management. Treatment options included oral glucocorticoids and biologic agents (etanercept, anakinra). Medical and pharmaceutical records were reviewed, and a bibliographic research was made to establish the state of the art treatment of TRAPS. UpToDate, Pubmed and the Cochrane Library were consulted, finding little information on this very rare medical condition.

Results Our patient was a 7 year old boy who presented with recurrent febrile episodes, accompanied by abdominal pain and periorbital eczema. There was no infectious focus. Laboratory data showed elevated inflammatory markers. The rheumatologist suspected an autoimmune syndrome rather than an autoinflammatory disease. Lack of autoantibodies and a genetic diagnosis confirmed TRAPS.

Initial treatment was oral prednisone, with a response similar to NSAIDs. Due to persistence of symptomatology, the clinician indicated etanercept, which achieved a partial response but had to be interrupted because of respiratory related sepsis. Afterwards, this biologic was reintroduced with low doses of prednisone. Over the following months the patient relapsed, and anakinra was prescribed instead of etanercept. Anakinra treatment showed satisfactory results, achieving symptomatology control and normalisation of laboratory parameters with no remarkable safety concerns.

Conclusion and relevance We have presented the case of a patient refractory to anti-TNF treatment who experienced dramatic improvement with the recombinant human IL-1 receptor antagonist anakinra. There are only a few cases published on this subject, and our experience supports the evidence that anakinra can be considered a firstline treatment for TRAPS due to its efficacy and lack of adverse reactions.

\section{REFERENCES AND/OR ACKNOWLEDGEMENTS}

No conflict of interest.

\section{CPS-131 EFFECTIVENESS OF NUSINERSEN IN PAEDIATRIC PATIENTS SMA1 AND SMA2}

${ }^{1} \mathrm{~GB}$ Ortenzi ${ }^{*},{ }^{2} \mathrm{G}$ Palmas, ${ }^{1} \mathrm{E}$ Andresciani, ${ }^{1} \mathrm{AMF}$ Garzone, ${ }^{3} \mathrm{MA}$ Berardi, ${ }^{4} \mathrm{~S}$ Siliquini, ${ }^{4} \mathrm{~L}$ Porfiri, ${ }^{1} \mathrm{MS}$ Demeo, 'S Guglielmi, ${ }^{3} \mathrm{C}$ Polidori, ${ }^{1} \mathrm{~A}$ Pompilio. ${ }^{1} \mathrm{AO}$ Ospedali Riunti Di Ancona, Sod Farmacia, Ancona, Italy; ${ }^{2}$ AO Ospedali Riuniti Ancona, Sod Clinica Pediatrica, Ancona, Italy; ${ }^{3}$ Università Degli Studi Di Camerino, Scuola Di Scienze Del Farmaco E Dei Prodotti Della Salute' Facoltà Di Farmacia, Camerino, Italy; ${ }^{4} \mathrm{AO}$ Ospedaliriunti Ancona, Sod Neuropsichiatria Infantile, Ancona, Italy

10.1136/ejhpharm-2020-eahpconf.232

Background and importance Nusinersen is an innovative drug given by intrathecal injection and used to treat $5 \mathrm{q}$-spinal muscular atrophy (SMA), a severe neuromuscular disorder due to a defect in the survival motor neuron 1 (SMN1) gene. This antisense oligonucleotide drug modifies RNA splicing of the SMN2 gene, thus increasing the production of full length $\mathrm{SMN}$ protein. The first dose, given as soon as possible after the diagnosis, should be followed by three more doses after 2, 4 and 9 (L1, L2, L3, L4) weeks and one dose every 4 months (M1, M2, M3...) thereafter.

Aim and objectives This study aims to describe the efficacy of nusinersen in terms of improvements in motor function in paediatric patients with SMA1 and SMA2.

Material and methods From February 2018, we collected data from 8 patients, 3 with SMA 1 and 5 with SMA 2, using specific neuromuscular functional tests: CHOP-INTEND, HINE and HFMSE

Results Results are expressed as points of increase (p) in motor function scores from baseline (or from the first score recorded in our centre*) to the score obtained at the time of the last injection for each patient.

SMA1 patients:

2 months old at the time of first injection (TFI): CHOPINTEND 8/64 to +38 p (M2); HINE $0 / 26$ to $+5 \mathrm{p}$ (M2).

3.3 years old TFI*: CHOP-INTEND 18/64 (M2) to $+16 \mathrm{p}$ (M6); HINE 2/26, stable at M6.

5.6 years old TFI*: CHOP-INTEND 1/64 (M2) to $-1 \mathrm{p}$ (M4); HINE 0/26 (M2) to +1p (M4), then suspended for absence of efficacy.

SMA2 patients:

1.2 years old TFI: CHOP-INTEND 59/64 to $-1 \mathrm{p}$ (M1).

3.4 years old TFI: CHOP-INTEND $41 / 64$ to +8 (M1), $+14 \mathrm{p}$ (M3); HFMSE $8 / 66$ to stable at M1 +4p (M3).

4.6 years old TFI: CHOP-INTEND $55 / 64$ to $+6 \mathrm{p}$ (M1) +7 (M2); HFMSE 22/66 to +3p (M1), +3p (M3).

8.5 years old TFI: CHOP-INTEND $42 / 64$ to $+5 \mathrm{p}$ (M1); HFMSE $17 / 66$ to $+10 \mathrm{p}$ (M1).

11.5 years old TFI: CHOP-INTEND $37 / 64$ to $+2 \mathrm{p}$ (M1); HFMSE 8/66 stable at M1.

Conclusion and relevance Our results showed an average increase of 4 points for CHOP-INTEND and 3.75 points for HFMSE in SMA2 patients, after 6 months (M1) of treatment. For SMA1 patients, it was not possible to evaluate the average trend for CHOP-INTEND and HINE scores after 6 months of treatment because two patients started nusinersen in other hospitals (motor scores at L1-M1 not available). A longer follow-up and data from other parameters, such as swallowing and respiratory function, are important to better understand the overall efficacy of nusinersen.

\section{REFERENCES AND/OR ACKNOWLEDGEMENTS}

No conflict of interest.

\section{CPS-132 INDIRECT TREATMENT COMPARISON OF ANTI- CALCITONIN GENE RELATED PEPTIDE PATHWAY ANTIBODIES IN CHRONIC MIGRAINE}

MDP Briceño Casado*, S Fenix-Caballero, MD Gil-Sierra, M Dominguez Cantero, EJ AlegreDel Rey. Hospital Universitario Puerto Real, Hospital Pharmacy, Cadiz, Spain

\subsection{6/ejhpharm-2020-eahpconf.233}

Background and importance Erenumab, fremanezumab, galcanezumab and eptinezumab are monoclonal antibodies targeting the calcitonin gene related peptide pathway (anti-CGRP), used as preventive treatment in chronic migraine (CM).

Aim and objectives To evaluate whether anti-CGRP drugs are equivalent therapeutic alternatives (ETA) in CM through an adjusted indirect treatment comparison (ITC).

Material and methods A bibliographic search of randomised clinical trials (RCTs) in Pubmed was performed (20 May 2019). Inclusion criteria: phase II/III RCTs of anti-CGPR with similar populations, follow-up duration and comparator treatments. CM was defined as $\geq 15$ headache days/month, of which $\geq 8$ were migraine days (event duration $\geq 4$ hours). Exclusion criteria: RCTs with different clinical CM context and other CM definitions. Efficacy end point was $\geq 50 \%$ reduction in migraine days/month (measured from the beginning of treatment to 12 weeks). An ITC was developed using 